\title{
The Need for Regulating a Bayesian Regulator*
}

\author{
SEMIH KORAY \\ Bilkent University \\ Department of Economics, Bilkent 06800, Ankara, Turkey \\ E-mail: ksemih@bilkent.edu.tr
}

ISMAIL SAGLAM

Bogazici University

Department of Economics, Bebek 34342, Istanbul, Turkey

E-mail:saglamis@boun.edu.tr

Bilkent University

Department of Economics, Bilkent 06800, Ankara, Turkey

E-mail:saglam@bilkent.edu.tr

\begin{abstract}
This paper analyzes the Baron and Myerson's (B-M) (Econometrica 50: 911-930 [1982]) scheme of monopoly regulation, a standard representative of Bayesian mechanisms. As is well known, the $\mathrm{B}-\mathrm{M}$ mechanism (and other related mechanisms) have as an explicit starting point the assumption that the regulator has an unchallenged prior belief about the cost function of the regulated monopolist.We analyze here the consequences resulting from the possibility that this prior belief may be subject to influence or manipulable. As we show in detail, under the B-M scheme, consumers and the regulated monopoly are highly sensitive to the regulator's prior belief about the (private) cost information of the monopolist. Therefore, if a regulator's beliefs are unaccountable to and unverifiable by a higher authority, the regulator has both the incentive and the possibility to change and/or misrepresent his prior belief when facing pressure or payoffs from interest groups representing consumers or the regulated firm. The results here show that the outcomes under a B-M mechanism favoring one or another interest group can vary over a wide spectrum. The results are consistent with capture theory and rent-seeking explanations of monopoly regulation and suggest the need to exercise care in using the insights and results of Bayesian regulatory theory to inform practice.
\end{abstract}

Key words: Bayesian mechanism, manipulable beliefs, monopoly, moral hazard, regulation

JEL Classifications: D42, D82, L51

* The authors thank Leonid Hurwicz, Murat Sertel, and seminar participants at Bilkent University and Bogazici University. The authors also thank the Editor and two anonymous referees of the Journal of Regulatory Economics for several useful comments and suggestions that have greatly improved the paper. The final revision of the paper was conducted while Saglam was visiting Bilkent University. Saglam also acknowledges the support of Turkish Academy of Sciences, in the framework of 'Distinguished Young Scientist Award Program' (TUBA-GEBIP-2004). The usual disclaimer applies. 


\section{Introduction}

In their famous paper Baron and Myerson (B-M) (1982) considered the problem of regulating a monopolistic firm whose costs are unknown to the regulator. In their setting marginal cost of production is constant and both the marginal and fixed cost are assumed to be affine functions of the firm's private parameter $\theta$, which lies in a real interval $\left[\theta_{0}, \theta_{1}\right]$. The regulator is assumed to know the form of the cost function and the interval $\left[\theta_{0}, \theta_{1}\right]$ to which the unknown parameter $\theta$ is known to belong. He also has a subjective prior belief about $\theta$, represented by a positive probability density function $f($.$) on \left[\theta_{0}, \theta_{1}\right]$. Informationally, the demand function is assumed to be known to the regulator as well as the firm.

The total social welfare is allowed to be any linear combination of consumers' and producer's surplus provided that the former is weighted at least as much as the latter. The B-M mechanism consists of four components: quantity, price, probability of the firm being allowed to produce and subsidy. The outcome which the B-M mechanism leads to depends properly upon the regulator's prior belief as well as the true value of the parameter $\theta$ in case consumers' and producer's surplus are not equally weighted. Here the subsidy paid to the firm is minimal for the total social welfare to be maximized under the constraint of both consumers' and producer's net gains being nonnegative. Although there are situations where this minimal amount is equal to the total consumers' surplus, there are many other situations which leave a positive portion of the consumers' surplus to the consumers themselves.

The B-M restrict themselves to incentive-compatible mechanisms which restriction, of course, leads to no loss of generality in view of the Revelation Principle (Gibbard 1973; Myerson 1979). Thus the true and reported values of $\theta$ coincide under this mechanism. In a static framework, not only is the true value of this cost parameter unchangeable but also its reported value is equal to its fixed true value. Thus once a producer with a particular value of $\theta$ is given, the outcome becomes entirely a function of the regulator's prior belief. This gives rise to a situation, where the regulator, the consumers and the producer may regard the density function $f$ as a "strategic variable". The positions of the consumers and the firm regarding this strategic variable differ, of course, due to the asymmetry with respect to the information about $\theta$. It turns out that both the producer and the consumers are extremely sensitive to the regulator's prior beliefs. Besides, the regulator possesses effective threat points against both the producer and the consumers.

We should note that in the static framework considered there are no past observations rendering certain probability distributions on $\left[\theta_{0}, \theta_{1}\right]$ superior to others. Moreover, there is no prior belief which maximizes actual social welfare no matter what the true value of $\theta$ is. Thus any positive probability density function represents a belief which is as "respectable" as any other. That is, the regulator's beliefs are entirely subjective and unaccountable to the public. There is no way of faulting a regulator for having a particular prior belief rather than another.

We imagine a situation where a subjective probability distribution about the value of $\theta$ is first being formed and then announced to the public prior to the 
implementation of the B-M mechanism. Some may object to this by saying that in the B-M framework the regulator's prior belief is not something which is to be formed and to be made public but it is something which is there and common knowledge from the outset and thus cannot be considered as a "strategic variable". A severe criticism to the common knowledge assumption as to the beliefs of a regulator is available in a recent work of Crew and Kleindorfer (2002), who argue that " ... the entire mechanism design literature, ..., is based in one way or another on assumptions like common knowledge that endow the regulator with information that he cannot have without a contested discovery process that always leaves him in a state far short of the level of information assumed in these theories. Common knowledge is the Achilles heel of mechanism design theory."

Here, it is very difficult for us to conceive of the regulator's prior belief as a kind of "law of nature" which is unchangeable, known, and fully agreed upon by the whole society. Such a strong assumption requires that the society is too well informed about its lack of information in a single shot without any past observations about the firm. Letting aside the epistemological question of what the source of the prior beliefs could be, we admit that our paper would be reduced to a vacuous one in that case. However, in the presence of a self-interest seeking agency who decides about what the prior belief should be, the assumption about the regulator's benevolence becomes a vacuous one. In that case, the floor gets open for manipulating prior beliefs which must be taken into account regarding the question of what outcome the B-M mechanism will lead to. At this point, we should acknowledge that the manipulability of the subjective prior beliefs of a Bayesian regulator that we make precise in this paper has in fact been conjectured and stressed for almost 20 years by many economists including Crew and Kleindorfer (1986), Vogelsang (1988, 2002, 2004), Koray and Sertel (1990) and Koray and Saglam (1999).

One of the explanations why a regulator with manipulable and unaccountable beliefs may misrepresent his genuine beliefs to the benefit of an interest group in the regulated environment is clearly income redistribution. As noted by Crew and Kleindorfer (2002), "Redistribution by regulation lacked the transparency and therefore the accountability of traditional methods of taxation and subsidy." Thus, a government who can perfectly control the manipulable beliefs of a regulator can redistribute the expected social surplus between the firm and the consumers in any way it wishes while simultaneously (pretending to be) maximizing a social objective function with the predetermined weights of the welfares of the two groups. Of course, generally governments do not decide on the form of the redistribution on their own. In our case, one can easily anticipate that once the regulated firm and the consumers notice that the beliefs of the regulator acting on behalf of the government are unaccountable to and unverifiable by the public, they may devote considerable efforts and resources to influence the regulator to make the aforementioned redistribution work in their direction.

So, our results are consistent with the rent-seeking explanation of monopoly regulation introduced by the pioneering work of Tullock (1967). If the regulator (or the governmental body that it represents) is dishonest, it may even demand 
some part of the social surplus as bribe from the firm and/or consumers during the rent-seeking process. However, the bribe such obtained, though totally unfair, may not be a (total) loss for the society as a whole, provided that the government spends or saves it in increasing the wealth of any group in the society including itself. Indeed, as pointed out by Tullock, there exists a much bigger potential danger of the rent-seeking process to the society (even in the presence of a honest and benevolent regulator). Since the firm and the consumers are both sensitive to the beliefs of the regulator, the resources invested by these two competing interest groups to influence in their favor the reported belief of a fully uninformed regulator with subject to influence or manipulable beliefs may simply offset each other to some extent and thus become purely wasteful from the standpoint of the society.

An alternative answer to the question why an honest regulator may manipulate his belief can be rooted in the "capture theory". According to the formulation of Posner (1974), this theory claims that over time regulatory agencies become captured (provide economic regulation according to the economic laws of supply and demand) by (to) the politically effective interest groups in the industry. So, the monopoly regulation problem we are dealing with also admits the possibility that either the regulated firm or the consumers' union captures the regulatory agency to dictate the prior belief the captured regulator should adopt.

We should note that all the results in this paper obtained within a Bayesian model of monopoly regulation straightforwardly extend to the general Bayesian framework of Guesnerie and Laffont (1984) which characterizes the complete solution to a class of principal-agency problems also admitting as special cases the income tax model of Mirrlees (1971), the quality choice model of the monopolist of Mussa and Rosen (1978) or Maskin and Riley (1984), and the model of regulating a natural monopolist of Baron and Myerson (1982).

The organization of the paper is as follows: Section 2 presents the B-M model of monopoly regulation as a well-known example of Bayesian mechanisms. Section 3 contains our results regarding how sensitive the different parties in the regulated environment are towards the admissible beliefs of the regulator. Finally, Section 4 concludes.

\section{A Bayesian Framework: Baron and Myerson's Optimal Regulatory Mechanism}

Baron and Myerson (1982) considers a model of regulating a natural monopoly with unknown costs. The firm (producer) has a cost function $C(q, \theta)$, where $q$ is the quantity produced and $\theta$ is a cost parameter that is unknown to the regulator. The firm's cost function is of the form

$$
C(q, \theta)=k+\theta q \quad \text { if } q>0 \quad \text { and } \quad C(0, \theta)=0
$$

where $\theta$ represents marginal cost and $k$ (a non-negative constant) represents setup cost of producing any positive output. The parameter $k$ and the form of the cost function are common knowledge. 
The unknown cost parameter $\theta$ is bounded within some interval $\left[\theta_{0}, \theta_{1}\right]$, where $\theta_{0}<\theta_{1}$. The regulator has prior belief $f(\theta)$ about the distribution of the true value of the cost parameter $\theta$ in the interval $\left[\theta_{0}, \theta_{1}\right]$. The density function $f(\theta)$ is positive and continuous over the interval $\left[\theta_{0}, \theta_{1}\right]$.

The inverse demand function is denoted by $P($.$) , and it is assumed to be nega-$ tively sloped. We also assume that the function $P($. $)$ is common knowledge.

The total value $V(q)$ of an output of quantity $q$ to consumers is the area under the inverse demand curve given by

$$
V(q)=\int_{0}^{q} P(\tilde{q}) d \tilde{q} .
$$

The consumers' surplus is $V(q)-P(q) q$.

A feasible regulatory policy $M=(r, p, q, s)$ consisting of four outcome functions is defined as follows: For any $\hat{\theta}$ in $\left[\theta_{0}, \theta_{1}\right], r(\hat{\theta})$ is the probability that the producer is permitted to operate after having reported $\hat{\theta}$ as its cost parameter. As a probability $r(\hat{\theta})$ should satisfy

$$
0 \leq r(\hat{\theta}) \leq 1 .
$$

Provided that the producer firm is permitted to operate, the regulated price $p(\hat{\theta})$ and the regulated output $q(\hat{\theta})$ should satisfy the inverse demand equation

$$
p(\hat{\theta})=P(q(\hat{\theta})) .
$$

Lastly, $s(\hat{\theta})=s^{*}(\hat{\theta}) r(\hat{\theta})$ is the expected value of the subsidy $s^{*}(\hat{\theta})$ paid to the producer if it is allowed to sell in the regulated market with probability $r(\hat{\theta})$ when it reports $\hat{\theta}$.

Let $\omega^{p}(\theta, \theta)$ be the (net) gain of the producer if it truthfully reveals its cost parameter $\theta$

$$
\omega^{p}(\theta, \theta)=[p(\theta) q(\theta)-\theta q(\theta)-k] r(\theta)+s(\theta)
$$

and let $\omega^{p}(\hat{\theta}, \theta)$ be the gain of the producer if it misreports its cost parameter as $\hat{\theta}$, while $\theta$ is the true cost parameter

$$
\omega^{p}(\hat{\theta}, \theta)=[p(\hat{\theta}) q(\hat{\theta})-\theta q(\hat{\theta})-k] r(\hat{\theta})+s(\hat{\theta}) .
$$

To make the producer report its true cost parameter $\theta$, the policy must be incentive-compatible:

$$
\omega^{p}(\theta) \equiv \omega^{p}(\theta, \theta)=\max _{\hat{\theta}} \omega^{p}(\hat{\theta}, \theta), \quad \forall \theta \in\left[\theta_{0}, \theta_{1}\right] .
$$

Finally the regulator must check that the regulatory policy is individually rational for a honest producer, that is

$$
\omega^{p}(\theta) \geq 0, \quad \forall \theta \in\left[\theta_{0}, \theta_{1}\right] .
$$


The feasibility conditions (3), (4), (7) and (8) ensure that the firm honestly reports its cost parameters.

At the cost report $\theta$ the consumers' (net) gain (the consumers' surplus net of the subsidy given to the firm) is denoted by $\omega^{c}(\theta)$, where

$$
\omega^{c}(\theta)=[V(q(\theta))-p(\theta) q(\theta)] r(\theta)-s(\theta) .
$$

The social welfare at the cost report $\theta$ is denoted by $\omega^{s}(\theta)$ and defined as a weighted sum of consumers' and producer's net gain:

$$
\omega^{s}(\theta)=\omega^{c}(\theta)+\alpha \omega^{p}(\theta),
$$

where $\alpha \in[0,1]$.

Denote the regulatory policy at the belief $f$ as $M_{f}=\left(r_{f}, p_{f}, q_{f}, s_{f}\right)$. Then given the firm's cost report $\theta$ and the regulator's prior belief $f$, the producer's gain, the consumers' gain, and the social welfare become $\omega_{f}^{p}(\theta), \omega_{f}^{c}(\theta)$ and $\omega_{f}^{s}(\theta)$, respectively.

The regulator's objective is to find a feasible regulatory policy that maximizes the expected social welfare

$$
W_{f}^{s}=\int_{\theta_{0}}^{\theta_{1}} \omega_{f}^{s}(\theta) f(\theta) d \theta .
$$

Now assume that $\theta+(1-\alpha) F(\theta) / f(\theta)$ is nondecreasing in $\theta \cdot{ }^{1}$ Then the optimal policy $\bar{M}_{f}=\left(\bar{r}_{f}, \bar{p}_{f}, \bar{q}_{f}, \bar{s}_{f}\right)$ to this welfare maximization problem is given by

$$
\bar{p}_{f}(\theta)=z_{\alpha, f}(\theta)
$$

with

$$
\begin{gathered}
z_{\alpha, f}(\theta)=\theta+(1-\alpha) \frac{F(\theta)}{f(\theta)}, \\
P\left(\bar{q}_{f}(\theta)\right)=\bar{p}_{f}(\theta), \\
\bar{r}_{f}(\theta)= \begin{cases}1 & \text { if } V\left(\bar{q}_{f}(\theta)\right)-\bar{p}_{f}(\theta) \bar{q}_{f}(\theta) \geq k, \\
0 & \text { if } V\left(\bar{q}_{f}(\theta)\right)-\bar{p}_{f}(\theta) \bar{q}_{f}(\theta)<k,\end{cases} \\
\bar{s}_{f}(\theta)=\left[\theta \bar{q}_{f}(\theta)+k-\bar{p}_{f}(\theta) \bar{q}_{f}(\theta)\right] \bar{r}_{f}(\theta)+\int_{\theta}^{\theta_{1}} \bar{r}_{f}(\tilde{\theta}) \bar{q}_{f}(\tilde{\theta}) d \tilde{\theta} .
\end{gathered}
$$

1 In fact, B-M does not need this sufficiency assumption on the beliefs that we solely make to simplify the analysis. B-M characterizes the optimal regulatory policy irrespective of the shape of the term $F(\theta) / f(\theta)$. 
As it is clear from (10) to (12), the optimal regulated price is higher than the marginal cost price unless $\alpha=1$, i.e. the consumers' and the producer's gains are equally weighted in the social welfare. In order to understand why this price schedule is optimal, we insert the optimal subsidy in (14) into (5) to obtain the producer's gain

$$
\bar{\omega}_{f}^{p}(\theta)=\int_{\theta}^{\theta_{1}} \bar{r}_{f}(\tilde{\theta}) \bar{q}_{f}(\tilde{\theta}) d \tilde{\theta} .
$$

We observe that the producer's gain is increasing in $\bar{q}_{f}$, the output produced. Moreover, rewriting the social welfare at the report $\theta$ as

$$
\bar{\omega}_{f}^{s}(\theta)=\left[V\left(\bar{q}_{f}(\theta)\right)-C\left(\bar{q}_{f}, \theta\right)\right] \bar{r}_{f}(\theta)-(1-\alpha) \bar{\omega}_{f}^{p}(\theta)
$$

we note the tradeoff between the efficiency and the incentives, which should be given to the producer. So, in order to limit the information rents of the producer, the optimal price is set above the marginal cost.

Equation (13) shows that the producer is allowed to produce only if the consumers' surplus at the reported cost is sufficiently high to cover the adjusted setup cost of production. Finally, we note that the optimal subsidy in (14) is composed of two parts: The integral part (given the other part) guarantees truthful revelation while the nonintegral part ensures that the producer will not make losses when he is allowed to produce.

\section{Preferences on Beliefs}

The optimal regulatory policy presented in Section 2 depends upon the prior belief of the regulator about the unknown cost parameter. This prior belief is assumed to be common knowledge, i.e. it is publicly known to both the consumers and the regulated producer. Here, what is restrictive is not that the regulator's announced belief is commonly shared by the society. Nowadays, extensively used public transmission and communication tools enable a credible regulator to make the public believe whatever he believes. Indeed, it is this very power of the regulator that is quite disturbing as the regulator can instead make the public believe whatever he pretends to believe. This may be a stronger case if the belief of the regulator does not derive from an objective structure or some past observations about the cost.

When the belief of the regulator reflects his subjective evaluations and hence is not completely verifiable by the society, the outcome of the regulatory mechanism is not predictable. The regulator with a manipulable belief becomes open to influence by the interest groups, the producer or the consumers, who may engage in costly rent-seeking activity to sway the regulator to their point of view. Below, we will make explicit how sensitive the welfares of these rent-seeking groups are to the regulator's prior belief.

We consider a Borel field $\mathcal{T}$ on the type space $\left[\theta_{0}, \theta_{1}\right]$ and regard the subset $\mathcal{A}$ of probability measures on $\mathcal{T}$ with densities that are strictly positive at each element 
of $\left[\theta_{0}, \theta_{1}\right]$ as the set of admissible prior beliefs for the regulator. Obviously, the set $\mathcal{A}$ which the regulator may choose his belief from is a rich continuum.

In the set of probability measures $\mathcal{T}$ there exists another continuum, so known as Dirac delta functions, which are of particular interest for our analysis.

Definition 1: The regulator completely knows that $\theta=\hat{\theta}$ if he has the belief represented by the Dirac delta function $\delta(\theta-\hat{\theta})$, which is defined as

$$
\delta(\theta-\hat{\theta})=0 \quad \text { if } \theta \neq \hat{\theta}, \quad \text { and } \quad \int_{\theta_{0}}^{\theta_{1}} \delta(\theta-\hat{\theta}) d \theta=1 .
$$

Notice that $\delta(\theta-\hat{\theta}) \notin \mathcal{A}$, i.e. the Dirac delta function is not an admissible belief since it is not a probability density function. Indeed, if the regulator was allowed to have as his belief a probability mass function (Dirac delta function) then the need for Bayesian regulation would cease to exist. However, one should still pay attention to the probability mass functions since in the continuum of admissible beliefs there exist ones that are arbitrarily close to $\delta(\theta-\hat{\theta})$ for all values of $\hat{\theta}$ in $\left[\theta_{0}, \theta_{1}\right]$. One can naturally ask how the outcome of the $\mathbf{B}-\mathbf{M}$ mechanism would change under beliefs which are arbitrarily close to functions representing the truth. To answer this question, we should first find out how the several parties in the economy rank different beliefs.

We index the set of representative agents for the three interest groups- the society, the consumers, and the producer in the regulated economy - by $\{s, c, p\}$.

Definition 2: Let $f, f^{\prime} \in \mathcal{A}$. The belief $f$ is preferred to $f^{\prime}$ by the representative agent $i$ under the optimal regulatory policy $\bar{M}_{f}$ if there exists $\left[\theta_{0}^{\prime}, \theta_{1}^{\prime}\right] \subset\left[\theta_{0}, \theta_{1}\right]$ such that $\bar{\omega}_{f}^{i}(\theta) \geq \bar{\omega}_{f^{\prime}}^{i}(\theta)$ for all $\theta \in\left[\theta_{0}, \theta_{1}\right]$ and $\bar{\omega}_{f}^{i}(\theta)>\bar{\omega}_{f^{\prime}}^{i}(\theta)$ for all $\theta \in\left[\theta_{0}^{\prime}, \theta_{1}^{\prime}\right]$.

Our first result below considers whether there is a most preferred belief in the view of the consumers and the society.

Proposition 1: Let $f \in \mathcal{A}$. Under the optimal regulatory policy $\bar{M}_{f}$ no belief in $\mathcal{A}$ is preferred to $f$ by the society or the consumers.

Proof: Suppose there exists $f^{\prime} \in \mathcal{A}$ that is preferred to $f$ by agent $s$ (representing the society) under the mechanism $\bar{M}_{f}$. Then $\int_{\theta_{0}}^{\theta_{1}} \bar{\omega}_{f^{\prime}}^{s}(\theta) f(\theta) d \theta>\int_{\theta_{0}}^{\theta_{1}} \bar{\omega}_{f}^{s}(\theta) f(\theta) d \theta$ implying that $\bar{M}_{f}$ is not optimal, which is a contradiction. That the consumers do not prefer $f^{\prime}$ to $f$ follows from the fact that $\bar{\omega}^{s}=\bar{\omega}^{c}$ for $\alpha=0$.

We will show that on the producer's side the situation seems to differ. The net gain (the information rent) accruing to the producer is extremely sensitive to the regulator's belief through the term $F(\theta) / f(\theta)$, so known as the "inverse of the reverse hazard rate". Now, we introduce a well-known dominance of beliefs. 
Definition 3: Consider two beliefs $f_{1}, f_{2} \in \mathcal{T}$. The belief $f_{1}$ stochastically dominates (in the inverse of the reverse hazard rate) $f_{2}$ if there exists $\left[\theta_{0}^{\prime}, \theta_{1}^{\prime}\right] \subset\left[\theta_{0}, \theta_{1}\right]$ such that $F_{1}(\theta) / f_{1}(\theta) \leq F_{2}(\theta) / f_{2}(\theta)$ for all $\theta \in\left[\theta_{0}, \theta_{1}\right]$ and $F_{1}(\theta) / f_{1}(\theta)<F_{2}(\theta) / f_{2}(\theta)$ for all $\theta \in\left[\theta_{0}^{\prime}, \theta_{1}^{\prime}\right]$.

We can state the following relation between our stochastic dominance concept and preferences of the monopoly over the beliefs.

Proposition 2: Let $f_{1}, f_{2} \in \mathcal{A}$ be such that $z_{\alpha, f_{1}}(\theta), z_{\alpha, f_{2}}(\theta)$ are non-decreasing in $\theta$ and $f_{1}$ stochastically dominates $f_{2}$. Then the producer prefers $f_{1}$ to $f_{2}$.

Proof: See Appendix A.

Proposition 3 shows that there are admissible beliefs which lead to outcomes arbitrarily close to the most preferred outcome for the producer.

Proposition 3: There exists a sequence of beliefs $\left\{f_{1}, f_{2}, \ldots, f_{\infty}\right\}$ such that $f_{j} \in \mathcal{A}$, $z_{\alpha, f_{j}}(\theta)$ is non-decreasing in $\theta$ for all $j$, and $\lim _{j \rightarrow \infty} f_{j}(\theta)=\delta\left(\theta-\theta_{1}\right)$. Then

$$
\lim _{j \rightarrow \infty} \bar{\omega}_{f_{j}}^{p}(\theta)=\sup _{f \in \mathcal{A}} \bar{\omega}_{f}^{p}(\theta), \quad \text { for all } \theta \in\left[\theta_{0}, \theta_{1}\right] \text {. }
$$

Proof: See Appendix A.

A sequence of beliefs admitted by Proposition 3 (as its proof suggests) is $\left\{f_{j}^{\epsilon}(\theta)\right\}_{j=1}^{\infty}$, where $\epsilon \in(0,1)$ and

$$
f_{j}^{\epsilon}(\theta)=\frac{1 / \epsilon^{j} \exp \left(\theta / \epsilon^{j}\right)}{\exp \left(\theta_{1} / \epsilon^{j}\right)-\exp \left(\theta_{0} / \epsilon^{j}\right)} .
$$

The limit of this sequence is $\delta\left(\theta-\theta_{1}\right)$, an inadmissible belief, which stochastically dominates any admissible belief. So, the above sequence yields in the limit to the producer a gain arbitrarily close to the supremum of $\bar{w}^{p}(\theta)$ for its cost report $\theta$. That is, the producer gains most if the regulator believes or pretends to believe that the producer is almost the least efficient type.

Although no belief is ex-post superior to another one from the viewpoint of either the consumers or the society for all realizations of the cost parameter, the below result establishes that by a suitable choice of the regulator's belief the social welfare and consumers' gain can be made arbitrarily close to zero unless the firm has the most efficient cost parameter $\theta_{0}$. Besides, under such a belief if $\theta=\theta_{0}$ then both the society and the consumers gain almost as much as what they would do under complete information. On the opposite side, for the same class of beliefs the information rent of the firm may be reduced down sufficiently close to zero for all realizations of the cost parameter, even for $\theta_{0}$.

Proposition 4: There exists a sequence of beliefs $\left\{f_{1}, f_{2}, \ldots, f_{\infty}\right\}$ such that $f_{j} \in \mathcal{A}$, $z_{\alpha, f_{j}}(\theta)$ is non-decreasing in $\theta$ for all $j$, and $\lim _{j \rightarrow \infty} f_{j}(\theta)=\delta\left(\theta-\theta_{0}\right)$. Then 
(i) for all $\theta \in\left[\theta_{0}, \theta_{1}\right]$

$$
\lim _{j \rightarrow \infty} \bar{\omega}_{f_{j}}^{p}(\theta)=\inf _{f \in \mathcal{A}} \bar{\omega}_{f}^{p}(\theta)
$$

(ii) for all $i$ in $\{s, c\}$

$$
\lim _{j \rightarrow \infty} \bar{\omega}_{f_{j}}^{i}(\theta)= \begin{cases}\inf _{f \in \mathcal{A}} \bar{\omega}_{f}^{i}(\theta) & \text { if } \theta \in\left(\theta_{0}, \theta_{1}\right], \\ \sup _{f \in \mathcal{A}} \bar{\omega}_{f}^{i}(\theta) & \text { if } \theta=\theta_{0} .\end{cases}
$$

Proof: See Appendix A.

Note from the proof of the above proposition that the sequence of beliefs $\left\{g_{j}^{\epsilon}(\theta)\right\}_{j=1}^{\infty}$ where $\epsilon \in(0,1)$ and

$$
g_{j}^{\epsilon}(\theta)=\frac{1 / \epsilon^{j} \exp \left(-\theta / \epsilon^{j}\right)}{\exp \left(-\theta_{0} / \epsilon^{j}\right)-\exp \left(-\theta_{1} / \epsilon^{j}\right)}
$$

converges to $\delta\left(\theta-\theta_{0}\right)$, the truth about $\theta_{0}$. One can show that this limiting (and inadmissible) belief is stochastically dominated by any admissible belief. It follows that if $\theta \neq \theta_{0}$, the producer, the consumers, and the society as a whole can be made to gain almost nil when the regulator pretends to believe that the producer is almost the most efficient type.

When the objective of the consumers changes from maximizing their ex-post gain to maximizing their ex-ante gain over the admissible set of preferences, we obtain another interesting result. First notice that among all social welfare functions the consumers most prefer the one which assigns zero weight to the producer's gain ( $\alpha=0$ case). One then wonders whether for every given belief $f \in \mathcal{A}$ of the regulator there exists another belief $f_{\alpha} \in \mathcal{A}$ such that the expected value under the belief $f_{\alpha}$ of the social welfare function that assigns the weight $\alpha$ to the producer's gain is the same as the expected consumers' gain under the regulator's original belief $f$.

To answer this question, we note that for any belief $f \in \mathcal{A}$ such that $F(\theta) / f(\theta)$ is non-decreasing, the expected social welfare at the optimal regulated output is

$$
\int_{\theta_{0}}^{\theta_{1}}\left[V\left(\bar{q}_{f}(\theta)\right)-C\left(\bar{q}_{f}, z_{\alpha, f}(\theta)\right)\right] \bar{r}_{f}(\theta) f(\theta) d \theta-(1-\alpha) \bar{\omega}_{f}^{p}\left(\theta_{1}\right),
$$

where $\bar{s}\left(\theta_{1}\right)$ can be chosen so as to make $\bar{\omega}_{f}^{p}\left(\theta_{1}\right)=0$. So, the expected consumers' gain under the belief $f$ is equal to the expected social welfare under the belief $f_{\alpha}$ for a given $\alpha \in[0,1]$ if $z_{\alpha, f_{\alpha}}=z_{0, f}$ or

$$
(1-\alpha) \frac{F_{\alpha}(\theta)}{f_{\alpha}(\theta)}=\frac{F(\theta)}{f(\theta)} .
$$

Rearranging the above equality yields

$$
\frac{f_{\alpha}(\theta)}{F_{\alpha}(\theta)}=(1-\alpha) \frac{f(\theta)}{F(\theta)}
$$


which implies $F_{\alpha}(\theta)=F(\theta)^{1-\alpha}$ and finally

$$
f_{\alpha}(\theta)=(1-\alpha) F(\theta)^{-\alpha} f(\theta) .
$$

We can now formally state the above findings.

Proposition 5: For any belief $f \in \mathcal{A}$ such that $F(\theta) / f(\theta)$ is non-decreasing and weight parameter $\alpha \in[0,1]$, consider the belief $f_{\alpha}=(1-\alpha) F(\theta)^{-\alpha} f(\theta)$. We have

$$
\int_{\theta_{0}}^{\theta_{1}} \bar{\omega}_{f_{\alpha}}^{s}(\theta) f_{\alpha}(\theta) d \theta=\int_{\theta_{0}}^{\theta_{1}} \bar{\omega}_{f}^{c}(\theta) f(\theta) d \theta
$$

The above results indicate that the regulator can, by a suitable choice of beliefs, redistribute the expected social surplus between the firm and the consumers according to the wishes of the government that it represents, while at the same time seeming to maximize the social objective function along with the weights of the welfares of the firm and the consumers predetermined by the legislation. It may even be the case that the regulator is "captured" by either the firm or the consumers and simply adopts the most desirable belief from the viewpoint of the interest group that it implicitly represents.

Even if the regulator does not have any ultimate goal of redistributing the social welfare or he is not wholly captured by any particular group in the regulated environment, one cannot deny the clear fact that the sensitivity of all the interest groups in the society to the regulator's manipulable beliefs renders the regulator open to influence by rent-seekers, including the consumers' union and the regulated firm. Moreover, the regulator might himself be a rent-seeker in this process if he demands/receives bribe from the regulated firm or the consumers in return for manipulating his beliefs. Here one should note that any belief of the regulator in the set $\mathcal{A}$ is equally admissible (and hence bribing is proved only with "corpus delicti", when the person attempting to bribe is caught on the site at the moment of offering or receiving the money). ${ }^{2}$ The regulator preferably takes the bribe before he announced his prior belief and the monopolist reported its cost parameter, thus the bribe paid by the firm or consumers does not change the form of the optimal regulatory policy. However, with bribery taking place the social welfare reported to the public decreases as the regulator's gain from manipulation has no positive weight assigned in the social welfare function.

Here, we find ourselves faced with a bargaining problem under asymmetric information. The firm reports its cost parameter $\theta$ after the regulator announced

2 Here we should also note that prohibiting certain beliefs to be owned by the regulator does not cure manipulation, because for any belief in the dense set $\mathcal{A}$, there exists another one which yields almost the same regulatory outcome. 
his belief, thus the regulator does not know the actual range of the bribe that he can extract from the regulated agents. The firm may decline to bribe if what the regulator demands from the firm (the consumers) is too high with respect to the producer's gain (the consumers' gain). The regulator if he commits to his threat would then announce the worst belief from the viewpoint of the firm (the consumers) so that the firm would produce (the consumers would buy) nil almost at any marginal cost. Such a case, of course, is not desirable for either the society or the regulator. All in all, one can regretfully say that "the fish stinks from the head down". The presence of a regulator with manipulable beliefs renders the regulatory mechanism prone to the rent-seeking of the regulator as well, and the associated 'rent-seeking solution', whose characterization is outside the scope of this paper, will, in general, differ from what the $\mathrm{B}-\mathrm{M}$ regulatory mechanism predicts.

A final remark to make is that both B-M results and the results that our analysis lead to strongly depend upon the asymmetry of information about the true value of the cost parameter. The firm is assumed to have complete information about it whereas neither the regulator nor the consumers are allowed to do so. So, we only compare the outcomes under the above informational structure with those under incomplete information from the viewpoints of all the parties involved, i.e., the consumers, the monopolistic firm and the society as a whole. This comparison naturally leads to the result that the uninformed parties - the consumers and the society as a whole - prefer complete information to incomplete while the firm prefers to keep its cost characteristics as private information until the regulator's prior belief is announced and thus becomes binding. This situation, of course, creates incentives for the uninformed parties to gain more information about the firm's cost parameter at the stage prior to the enforcement of the mechanism if they can do so. ${ }^{3}$

\section{Conclusions}

Dealing with the B-M scheme of monopoly regulation, a well-known representative of Bayesian mechanisms, we have established that both the regulated firm and the consumers are sensitive to the regulator's prior belief about the firm's private cost parameter. Although there is neither a best nor a worst prior belief for the firm due to the structure of the class of functions to which prior beliefs are supposed to belong, it turns out that there are beliefs, which lead to outcomes arbitrarily close to the most preferred or to the least preferred outcome for the firm, rendering the firm extremely sensitive to what the prior belief of the regulator is.

3 Koray and Saglam (2004) examine whether the regulated agent may have any incentive to signal his private information so as to change the regulator's belief. An interesting result they obtain is that more information about the regulated agent is not always desired and valued by the society or the principal (i.e. consumers in the monopoly regulation problem). 
On the other hand, from the consumers' viewpoint there is no prior belief dominating others, that is leading to an actual consumers' surplus which is higher than those resulting from other prior beliefs no matter what the true value of the firm's cost parameter is. Nevertheless, for every prior belief of the regulator there exists another one, such that a regulator possessing that prior belief would end up maximizing the expected value of the consumers' surplus evaluated according to the former belief under the B-M mechanism. Thus consumers who somehow know and agree with the regulator's prior belief would still wish that the regulator behaved as if he had the latter prior belief. Moreover, the regulator possesses admissible beliefs inducing values of net consumers' gain as close to nil as we wish irrespective of the value of $\theta$. All in all, the consumers are not impartial towards the regulator's prior belief either.

Our findings are consistent with both capture theory and rent-seeking explanations of monopoly regulation and suggest the need to exercise care in using the insights and results of Bayesian regulatory theory to inform practice.

Our results on Bayesian mechanisms also contribute to the ongoing debate between Bayesian and non-Bayesian approach in regulation. Non-Bayesian mechanisms are dynamic price adjustment rules that use the observable and verifiable data on the regulated industry. Among such mechanisms (regulating a monopoly), the pioneering scheme of Loeb and Magat (1979) entitles the firm to the whole social surplus and thus ensures the first-best optimum in a single period unlike the other non-Bayesian mechanisms, though at the expense of an utter inequity in the society. The Incremental Surplus Subsidy (ISS) scheme by Sappington and Sibley (1988) refines the Loeb and Magat scheme by taxing away the surplus the firm receives after one period. The outcome of this mechanism converges to the first-best optimum in the second period. The adjustment mechanism proposed by Finsinger and Vogelsang ( $\mathrm{F}-\mathrm{V})$ (1982) uses an observable approximation to the consumer surplus change offered to the regulated firm. The F-V scheme, which is otherwise quite similar to the ISS scheme, only leads to a convergence to the firstbest optimum in the long-run. The $\mathrm{F}-\mathrm{V}$ scheme gives the firm incentives to incur purely wasteful expenditures in early periods, while this serious drawback of the scheme is overcome in a refinement due to Hagerman (1990) with the introduction of service payments. Generally, the pricing rules that non-Bayesian mechanisms suggest do not maximize a well-defined multi-period social welfare function, and the convergence under these mechanisms to the optimum of the associated singleshot regulation problem is not immediate. One can nevertheless evaluate the relative welfare performance of non-Bayesian mechanisms as in the work of Lyon (1996).

While the Bayesian approach in regulation always leads to the (incomplete information) optimum, it has been criticized for its unrealistic Bayesian description of asymmetric information. Indeed, what this paper makes precise is an already known conjecture that the beliefs of a Bayesian regulator, hence the outcome they lead to, are manipulable. Along the same direction, Crew and Kleindorfer (2002) $(\mathrm{C}-\mathrm{K})$ also criticize the Bayesian approach for it does not make explicit the derivation of the probability density function representing the regulator's prior belief and 
for it conceives the regulator's prior belief as common knowledge. Both $\mathrm{C}-\mathrm{K}$ and Vogelsang (2004) make a further criticism of the Bayesian approach in its granting an unrealistic ability to the regulator to concede some (theoretically unavoidable) information rents to the regulated firm(s). The $\mathrm{C}-\mathrm{K}$ also criticizes another unquestioned condition for the efficiency of the Bayesian approach, namely the commitment of the regulator to the original regulatory scheme guaranteeing non-negative information rents to the regulated agent after revealing his private information.

Besides its undesired aspects, the Bayesian approach in regulation also has some qualitative results that are discussed at length in Vogelsang (2004). An invaluable insight that we have learned in the last two decades is that information rent as well as the cost-reducing effort of the regulated firm should be increasing in its innate efficiency. An equally strong recommendation is that increasing regulatory commitment is desirable and renegotiation of the information rents promised to the firm is generally not optimal. Considering the shortcomings of the Bayesian approach noted in this paper and the recognized efficiency drawbacks of existing implementable non-Bayesian mechanisms regulatory economists are left with something of a quandary. Perhaps the view expressed by Vogelsang (2004) represents the appropriate middle-ground to which these results naturally lead, namely that "the results from the Bayesian literature could determine the design of non-Bayesian mechanisms and the choice among several non-Bayesian mechanisms."

\section{Appendix A}

Proof of Proposition 2: From (6), (14) and (15), the producer's gain can be written as

$$
\bar{\omega}_{f}^{p}(\theta)=\int_{\theta_{0}}^{\theta_{f}^{*}} \bar{q}_{f}(\tilde{\theta}) d \tilde{\theta}
$$

with $\theta_{f}^{*}=\max \left\{\theta \in\left[\theta_{0}, \theta_{1}\right] \mid \bar{r}_{f}(\theta)=1\right\}$, where

$$
\bar{r}_{f}(\theta)= \begin{cases}1 & \text { if } \Gamma_{f}(\theta) \geq 0, \\ 0 & \text { if } \Gamma_{f}(\theta)<0\end{cases}
$$

and $\Gamma_{f}(\theta)=V\left(\bar{q}_{f}(\theta)\right)-\bar{p}_{f}(\theta) \bar{q}_{f}(\theta)-k$. Differentiating $\Gamma_{f}(\theta)$ with respect to $z_{\alpha, f}(\theta)$ we get

$$
\frac{\partial \Gamma_{f}(\theta)}{\partial z_{\alpha, f}(\theta)}=-\bar{q}_{f}(\theta) \frac{\partial \bar{p}_{f}(\theta)}{\partial z_{\alpha, f}(\theta)}<0,
$$

noting $\bar{p}_{f}(\theta)=z_{\alpha, f}(\theta)$. Then using $z_{\alpha, f}=\theta+(1-\alpha) F(\theta) / f(\theta)$ we get

$$
\frac{\partial \Gamma_{f}(\theta)}{\partial \frac{F(\theta)}{f(\theta)}}=-(1-\alpha) \bar{q}_{f}(\theta) \leq 0 .
$$

So, $\theta_{f}^{*}$ is non-increasing in $F(\theta) / f(\theta)$. Therefore, $\omega_{f}^{p}(\theta)$ is non-increasing in $F(\theta) / f(\theta)$, and the producer prefers $f_{1}$ to $f_{2}$. 
Proof of Proposition 3: Consider the sequence $\left\{f_{j}^{\epsilon}(\theta)\right\}_{j=1}^{\infty}$, where $\epsilon \in(0,1)$ and

$$
f_{j}^{\epsilon}(\theta)=\frac{1 / \epsilon^{j} \exp \left(\theta / \epsilon^{j}\right)}{\exp \left(\theta_{1} / \epsilon^{j}\right)-\exp \left(\theta_{0} / \epsilon^{j}\right)} .
$$

We will show that the limit of this sequence, an inadmissible belief, stochastically dominates all admissible beliefs. First observe

$$
\frac{F_{j}^{\epsilon}(\theta)}{f_{j}^{\epsilon}(\theta)}=\epsilon^{j}\left(1-\exp \left(\frac{\theta_{0}-\theta}{\epsilon^{j}}\right)\right) .
$$

It follows that

$$
\lim _{j \rightarrow \infty} \bar{\omega}_{f_{j}^{\epsilon}}^{p}(\theta)=\sup _{f \in \mathcal{A}} \bar{\omega}_{f}^{p}(\theta), \quad \text { for all } \theta \in\left[\theta_{0}, \theta_{1}\right]
$$

since $\partial \bar{\omega}_{f}^{p}(\theta) / \partial z_{\alpha, f}(\theta) \leq 0$ for all $f \in \mathcal{A}, \lim _{j \rightarrow \infty} z_{\alpha, f_{j}^{\epsilon}}(\theta)=\lim _{j \rightarrow \infty} \theta+(1-\alpha)$ $F_{j}^{\epsilon}(\theta) / f_{j}^{\epsilon}(\theta)=\theta=\inf _{f \in \mathcal{A}} z_{\alpha, f}(\theta)$ for all $\theta \in\left[\theta_{0}, \theta_{1}\right)$ and $\bar{\omega}_{f}^{p}\left(\theta_{1}\right)=0$ for all $f \in \mathcal{A}$. Moreover,

$$
\begin{aligned}
\lim _{j \rightarrow \infty} f_{j}^{\epsilon}(\theta) & =\lim _{j \rightarrow \infty} \frac{1 / \epsilon^{j} \exp \left(\theta / \epsilon^{j}\right)}{\exp \left(\theta_{1} / \epsilon^{j}\right)-\exp \left(\theta_{0} / \epsilon^{j}\right)} \\
& =\lim _{h \rightarrow \infty} \frac{h \exp (\theta h)}{\exp \left(\theta_{1} h\right)-\exp \left(\theta_{0} h\right)} \\
& =\lim _{h \rightarrow \infty} \frac{h \exp \left(\left(\theta-\theta_{0}\right) h\right)}{\exp \left(\left(\theta_{1}-\theta_{0}\right) h\right)-1} \\
& =\lim _{h \rightarrow \infty} \frac{1+h\left(\theta-\theta_{0}\right)}{\exp \left(\left(\theta_{1}-\theta\right) h\right)\left(\theta_{1}-\theta_{0}\right)} \\
& =\lim _{h \rightarrow \infty} \frac{\theta-\theta_{0}}{\exp \left(\left(\theta_{1}-\theta\right) h\right)\left(\theta_{1}-\theta\right)\left(\theta_{1}-\theta_{0}\right)} \\
& =\delta\left(\theta-\theta_{1}\right)
\end{aligned}
$$

since we also have $F_{j}^{\epsilon}\left(\theta_{1}\right)-F_{j}^{\epsilon}\left(\theta_{0}\right)=1$ for all $j$.

Proof of Proposition 4: Consider the sequence $\left\{g_{j}^{\epsilon}(\theta)\right\}_{j=1}^{\infty}$, where $\epsilon \in(0,1)$ and

$$
g_{j}^{\epsilon}(\theta)=\frac{1 / \epsilon^{j} \exp \left(-\theta / \epsilon^{j}\right)}{\exp \left(-\theta_{0} / \epsilon^{j}\right)-\exp \left(-\theta_{1} / \epsilon^{j}\right)} .
$$


We will show that the limit of this sequence (an inadmissible belief) is stochastically dominated by any admissible belief. First observe

$$
\begin{aligned}
\lim _{j \rightarrow \infty} g_{\epsilon^{j}}(\theta) & =\lim _{j \rightarrow \infty} \frac{1 / \epsilon^{j} \exp \left(-\theta / \epsilon^{j}\right)}{\exp \left(-\theta_{0} / \epsilon^{j}\right)-\exp \left(-\theta_{1} / \epsilon^{j}\right)} \\
& =\lim _{h \rightarrow 0} \frac{1 / h}{\exp \left(\left(\theta-\theta_{0}\right) / h\right)-\exp \left(\left(\theta-\theta_{1}\right) / h\right)} \\
& =\lim _{h \rightarrow 0} \frac{1 / h}{\exp \left(\left(\theta-\theta_{0}\right) / h\right)} \\
& =\lim _{h \rightarrow 0} \frac{-1 / h^{2}}{-1 / h^{2}\left(\theta-\theta_{0}\right) \exp \left(\left(\theta-\theta_{0}\right) / h\right)} \\
& =\lim _{h \rightarrow 0} \frac{1}{\left(\theta-\theta_{0}\right) \exp \left(\left(\theta-\theta_{0}\right) / h\right)} \\
& =\delta\left(\theta-\theta_{0}\right)
\end{aligned}
$$

since we also have $G_{j}^{\epsilon}\left(\theta_{1}\right)-G_{j}^{\epsilon}\left(\theta_{0}\right)=1$ for all $j$.

Noting that

$$
\frac{G_{j}^{\epsilon}(\theta)}{g_{j}^{\epsilon}(\theta)}=\epsilon^{j}\left(\exp \left(\frac{\theta-\theta_{0}}{\epsilon^{j}}\right)-1\right)
$$

we obtain

$$
\lim _{j \rightarrow \infty} \bar{\omega}_{g_{j}^{\epsilon}}^{p}(\theta)=\inf _{f \in \mathcal{A}} \bar{\omega}_{f}^{p}(\theta), \quad \text { for all } \theta \in\left[\theta_{0}, \theta_{1}\right]
$$

since $\partial \bar{\omega}_{f}^{p}(\theta) / \partial z_{\alpha, f}(\theta) \leq 0$ for all $f \in \mathcal{A}, \lim _{j \rightarrow \infty} z_{\alpha, g_{j}^{\epsilon}}(\theta)=\lim _{j \rightarrow \infty} \theta+(1-\alpha)$ $G_{j}^{\epsilon}(\theta) / g_{j}^{\epsilon}(\theta)=\infty=\sup _{f \in \mathcal{A}} z_{\alpha, f}(\theta)$ for all $\theta \in\left(\theta_{0}, \theta_{1}\right]$. (Note that $\lim _{j \rightarrow \infty} \bar{q}_{g_{j}^{\epsilon}}(\theta)=0$ for all $\theta \in\left(\theta_{0}, \theta_{1}\right]$ also implies $\lim _{j \rightarrow \infty} \bar{\omega}_{g_{j}^{\epsilon}}^{p}\left(\theta_{0}\right)=0$.)

That $\lim _{j \rightarrow \infty} \bar{q}_{g_{j}^{\epsilon}}(\theta)=0$ for all $\theta \in\left(\theta_{0}, \theta_{1}\right]$ altogether with $\lim _{j \rightarrow \infty} \bar{q}_{g_{j}^{\epsilon}}\left(\theta_{0}\right)=$ $P^{-1}\left(\theta_{0}\right)$ proves part (ii) of the proposition.

\section{References}

Baron, D., and R. B. Myerson. 1982. "Regulating a Monopolist with Unknown Costs." Econometrica 50: 911-930.

Crew, M. A., and P. R. Kleindorfer. 1986. The Economics of Public Utility Regulation. Cambridge, MA: MIT Press.

Crew, M. A., and P. R. Kleindorfer. 2002. "Regulatory Economics: Twenty Years of Progress?" Journal of Regulatory Economics 21: 5-22.

Finsinger, J., and I. Vogelsang. 1982. "Performance Indices for Public Enterprises." In Public Enterprise in Less Developed Countries, edited by L. P. Jones. New York: Cambridge University Press.

Gibbard, A. 1973. "Manipulation of Voting Schemes: A General Result." Econometrica 41: 587-602.

Guesnerie, R., and J. J. Laffont. 1984. "A Complete Solution to a Class of Principal-Agent Problems with an Application to the Control of a Self-Managed Firm." Journal of Public Economics 25: 329-369. 
Hagerman, J. 1990. "Regulation by Price Adjustment." The RAND Journal of Economics 21: 72-82.

Koray, S., and M. R. Sertel. 1990. "Pretend-but-Perform Regulation and Limit Pricing." European Journal of Political Economy 6: 451-472.

Koray, S., and I. Saglam. 1999. "Bayesian Regulatory Mechanisms: Corruption and Learning." In Contemporary Economic Issues. Volume 4: Economic Behavior and Design, edited by M. Kaser and M. R. Sertel. London: MacMillan Press.

Koray, S., and I. Saglam. 2004. "Learning in Bayesian Regulatory Mechanisms." Mimeo.

Loeb, M., and W. A. Magat 1979. "A Decentralized Method for Utility Regulation." Journal of Law and Economics 22: 399-404.

Lyon, T. P. 1996. "Evaluating the Performance of Non-Bayesian Regulatory Mechanisms." Journal of Regulatory Economics 9: 41-60.

Maskin, E., and J. Riley. 1984. "Monopoly With Incomplete Information." The Rand Journal of Economics 15: 171-196.

Mirrlees, J. 1971. "An Exploration in the Theory of Optimum Income Taxation." Review of Economic Studies 38: 175-208.

Mussa, M., and S. Rosen. 1978. "Monopoly and Product Quality." Journal of Economic Theory 18: 301317.

Myerson, R. B. 1979. "Incentive Compatibility and the Bargaining Problem." Econometrica 47: 61-74.

Posner, R. A. 1974. "Theories of Economic Regulation." Bell Journal of Economics 5: 335-358.

Sappington, D. and D. Sibley. 1988. "Regulating Without Cost Information: The Incremental Surplus Subsidy Scheme." International Economic Review 29: 297-306.

Tullock, G. 1967. "The Welfare Costs of Tariffs, Monopolies, and Theft." Western Economic Journal 5: 224-232.

Vogelsang, I. 1988. "A Little Paradox in the Design of Regulatory Mechanisms." International Economic Review 29: 467-476.

Vogelsang, I. 2002. "Incentive Regulation and Competition in Public Utility Markets: A 20-Year Perspective." Journal of Regulatory Economics 22: 5-27.

Vogelsang, I. 2004. "Transmission Pricing and Performance-Based Regulation," presented at CarnegieMellon Conference on Electricity Transmission in Deregulated Markets: Challenges, Opportunities, and Necessary R\&D Agenda: Pittsburgh, December. 